\title{
Índice Orientador do Tratamento Sistêmico da Gravidez Ectópica Íntegira com Dose Única de Metotrexato
}

\author{
Index for the Systemic Treatment of Unruptured Ectopic Pregnancy with \\ a Single Dose of Methotrexate
}

Julio Elito Junior, André Reichmann, Mary N. Uchiyama, Luiz Camano

\begin{abstract}
RESUM0
Foi realizado estudo longitudinal em 42 pacientes com diagnóstico de gravidez ectópica integra, com o intuito de se elaborar um índice orientador do uso sistêmico de metotrexato em dose única $\left(50 \mathrm{mg} / \mathrm{m}^{2}\right)$ por via intramuscular. $O$ acompanhamento se fez através de dosagens de $\beta$-hCG (fração beta do hormônio gonadotrópico coriônico) realizadas no $1^{\circ}, 4^{\circ} e$ $7^{\circ}$ dias após o emprego do quimioterápico. Quando ocorreu queda de $15 \%$ ou mais nos títulos de $\beta$-hCG, apurados no $4^{\circ}$ e no $7^{\circ}$ dia, as pacientes receberam alta hospitalar $e$ seguimento ambulatorial com dosagens semanais de $\beta$-hCG até que se atingissem niveis inferiores a $5 \mathrm{mUI} / \mathrm{ml}$. Foi elaborado um indice orientador do tratamento sistêmico com metotrexato baseado nos seguintes parâmetros: (1) valores iniciais de $\beta$ - $h C G$; (2) aspecto da imagem à ultra-sonografia (hematossalpinge, anel tubário, embrião vivo); (3) maior diâmetro da massa anexial; (4) quantidade de líquido livre; (5) fluxo vascular medido por meio do doppler colorido. Cada parâmetro recebeu pontuação de 0 a 2. A nota zero significa elemento de mau prognóstico, a nota dois indica parâmetros favoráveis e a nota um, situações intermediárias. O índice de sucesso com dose única foi de 69,0\% (29/42 pacientes). A ultrasonografia transvaginal com doppler colorido foi realizada em 20 das 42 pacientes do estudo. Neste grupo de 20 pacientes o sucesso do tratamento ocorreu em 75,0\% dos casos (15/20). Entre as 22 pacientes que não foram avaliadas com doppler colorido a média das notas do indice nos casos de sucesso foi de 6,6, nas de insucesso 3,1. No grupo de pacientes avaliadas por doppler (20 pacientes) as médias foram de 7,9 (sucesso) e 4,2 (fracasso). No presente estudo a nota de corte foi estabelecida levando-se em conta o valor abaixo do qual o tratamento não foi efetivo e correspondeu a cinco, pois $93,75 \%$ das pacientes com nota superior a 5 evoluíram com sucesso (15/16), ao passo que notas inferiores ou iguais a cinco estiveram todas relacionadas com o fracasso do tratamento. $O$ índice orientador ajuda-nos a indicar os melhores casos para o tratamento medicamentoso. Não o aconselhamos, portanto, quando a nota for inferior ou igual a cinco; por outro lado, podemos predizer boa evolução do tratamento, quando a nota for superior a cinco.
\end{abstract}

PALAVRAS-CHAVE: Gravidez Ectópica. Metotrexato. Quimioterapia.

Disciplina de Obstetrícia da Universidade Federal de São Paulo

Departamento de Diagnóstico por Imagem da Universidade

Federal de São Paulo - EPM

Correspondência:

Julio Elito Junior

Endereço: Rua Sampaio Vidal, 824 - Jardim Paulistano

Cep: 01443-000 - São Paulo - SP

Tel.: 852-4769 
Introdução

Desde o surgimento do tratamento medicamentoso da gravidez ectópica (GE), em 1982, com Tanaka et al. ${ }^{19}$, este vem sendo empregada como uma alternativa terapêutica. Diversos autores têm estudado essa conduta, utilizando vários medicamentos e vias de administração diferentes, com índices de sucesso que oscilam entre 72,7 e $96,7 \%{ }^{5,8,9,10,16,18}$. Em razão dos danos causados pela ruptura tubária, quando ocorre a falha desta terapêutica medicamentosa, criou-se uma polêmica: qual o melhor procedimento terapêuticoo cirúrgico ou o medicamentoso?

Ao selecionar pacientes com GE íntegra para tratamento medicamentoso com sucesso evitamos os riscos anestésico-cirúrgicos, os custos são menores e o retorno às atividades mais rápido, além de preservarmos a capacidade reprodutiva. Por outro lado, quando ocorre fracasso desta terapêutica, temos uma situação adversa, com possibilidade de ruptura da GE, tornando necessário um procedimento de emergência, que na grande maioria dos casos, leva à opção de salpingectomia. Além disso, impõe-se, muitas vezes, a reposição sangüínea. Nesta situação questiona-se a realização da cirurgia conservadora ou mesmo o emprego da videolaparoscopia.

Para tentar responder a esta pergunta preparamos um índice com o intuito de selecionar um grupo de pacientes que venham a se beneficiar do tratamento medicamentoso. Na composição deste índice incluímos parâmetros: valores iniciais de $\beta$-hCG sérico, aspectos da imagem à ultrasonografia, diâmetro máximo da massa, presença de líquido livre na cavidade peritoneal e ultrasonografia (US) com “doppler” colorido.

\section{Pacientes e Métodos}

Este estudo longitudinal foi realizado na Disciplina de Obstetrícia da Universidade Federal de São Paulo - Escola Paulista de Medicina, quando foi padronizado o tratamento medicamentoso da GE integra.

Foram avaliadas 70 pacientes com diagnóstico de GE, atendidas no período de abril de 1994 a dezembro de 1996. Destas, 42 concordaram com o tratamento sistêmico com MTX (50 mg/m² IM), em outras 20 optamos pela conduta expectante (títulos de $\beta-\mathrm{hCG}$ em regressão) e 6 recusaram-se em participar dos protocolos, sendo submetidas ao tratamento cirúrgico. Houve dois casos de gravidez ectópica cervical para as quais indicamos tratamento local.

Para o diagnóstico precoce de GE íntegra, valorizamos os dados da história clínica e do exame físico, juntamente com os exames subsidiários: dosagem sérica de $\beta-\mathrm{hCG}$ e a US transvaginal. Acrescentamos a estes exames, a partir do $23^{\circ}$ caso, a US transvaginal com doppler colorido.

As dosagens da fração $\beta$ do hormônio coriônico gonadotrópico foram realizadas pelo método imunoenzimométrico (Aia-Pack bhCG, Tosoh Medics, INC.). A ultra-sonografia foi realizada com equipamento Atl UM-9 (Bothel, Wash) com transdutor de banda larga de 5-9 Mhz para via transvaginal. Após minucioso exame em escalas de cinza, utilizamos o doppler colorido para o mapeamento vascular do útero, ovários e eventuais massas anexiais, seguindo-se por último a análise espectral dos fluxos encontrados nos anexos e, quando necessário, da região periendometrial.

As massas anexiais foram caracterizadas conforme o seu aspecto ao US em: hematossalpinge, anel tubário e saco gestacional com embrião vivo. Em nosso estudo caracterizaremos cada um dos aspectos US de forma mais pormenorizada como descrito a seguir.

A imagem patognomônica da GE é a presença de saco gestacional ectópico, o qual contém o embrião e este apresenta batimentos cardíacos (Figura 1). Quanto ao anel tubário, (situações em que há saco gestacional e ausência de embrião vivo) podemos encontrar na US as seguintes situações:

- saco gestacional que pode apresentar embrião, porém sem atividade cardíaca (Figura 2); saco gestacional contendo apenas a vesícula vitelínica (Figura 3); saco gestacional vazio, sendo que este se apresenta hiperecogênico e tem forma de anel, sendo o seu centro anecóico (Figura 4).

A hematossalpinge tem o seguinte aspecto à ecografia: presença de massa sólida, com ecogenicidade complexa, apresentando áreas hiper e hipoecóicas irregulares (Figura 5). O plano de clivagem com o ovário foi avaliado para descartar a possibilidade de a massa ser de origem ovariana.

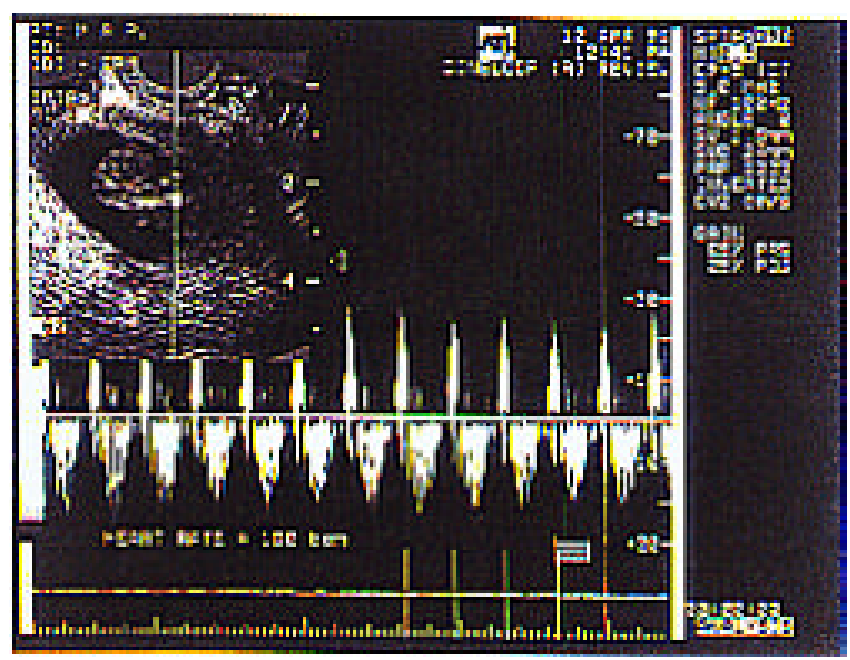

Figura 1 - Ultra-sonografia transvaginal demonstrando embrião com batimentos cardíacos com o recurso do doppler. O cursor foi colocado na área cardíaca do embrião demonstrando sua freqüência cardíaca. 


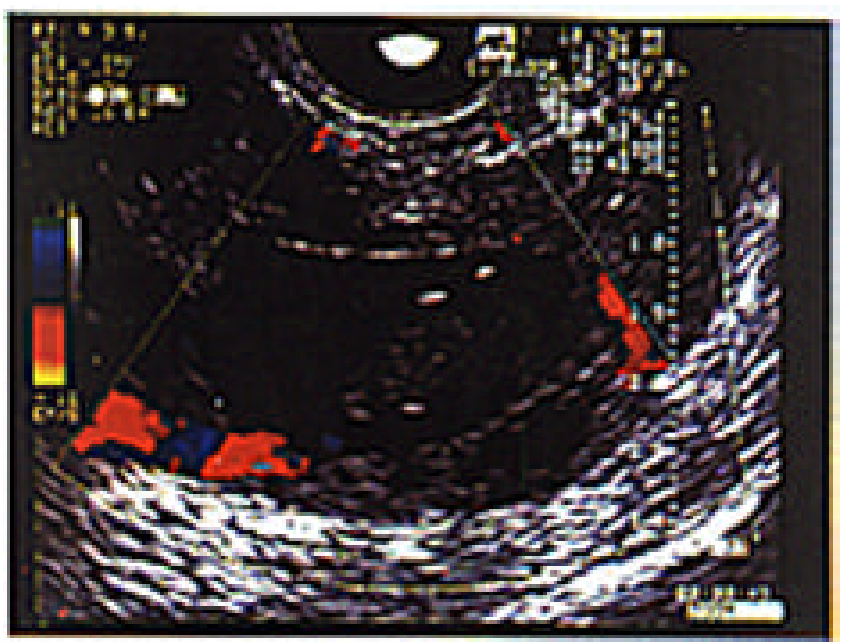

Figura 2 - Gravidez tubária íntegra com presença de embrião morto no interior do saco gestacional. Não se observa fluxo na área cardíaca.

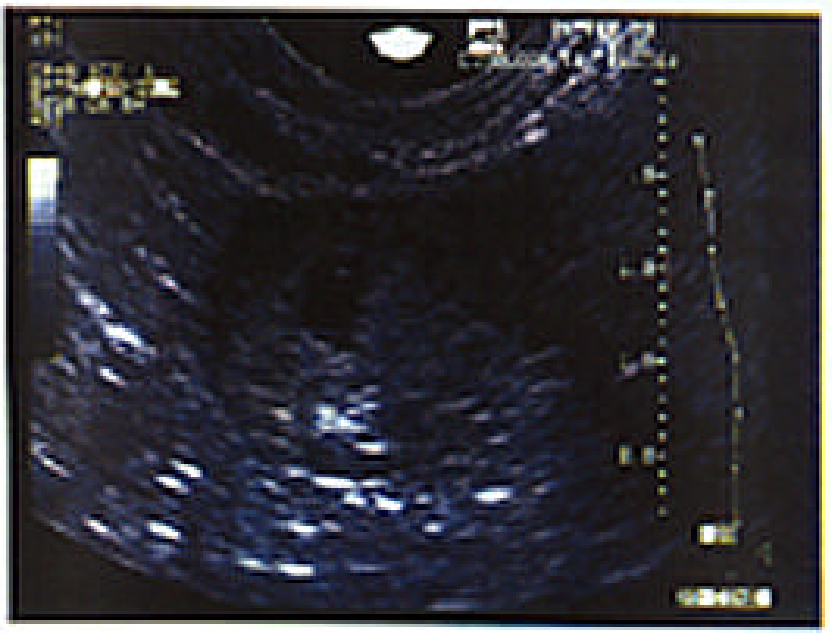

Figura 3 - Por meio de ultra-sonografia transvaginal foi possível visibilizar gravidez tubária íntegra com saco gestacional e no seu interior a presença de vesícula vitelínica.

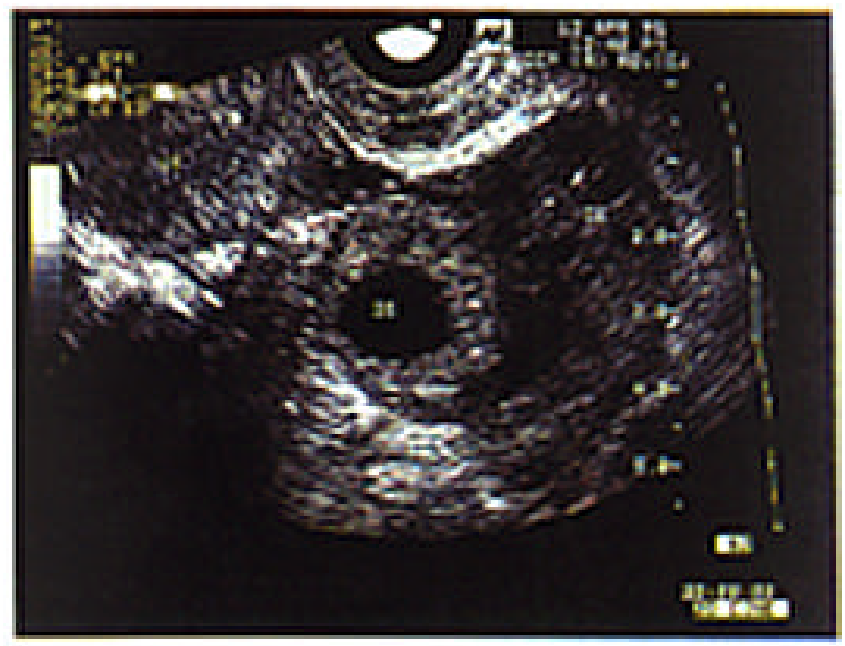

Figura 4 - Observa-se neste exame a presença do útero e do ovário esquerdo e entre eles encontra-se a gravidez tubária, neste caso apresentando-se com 0 aspecto de saco gestacional vazio

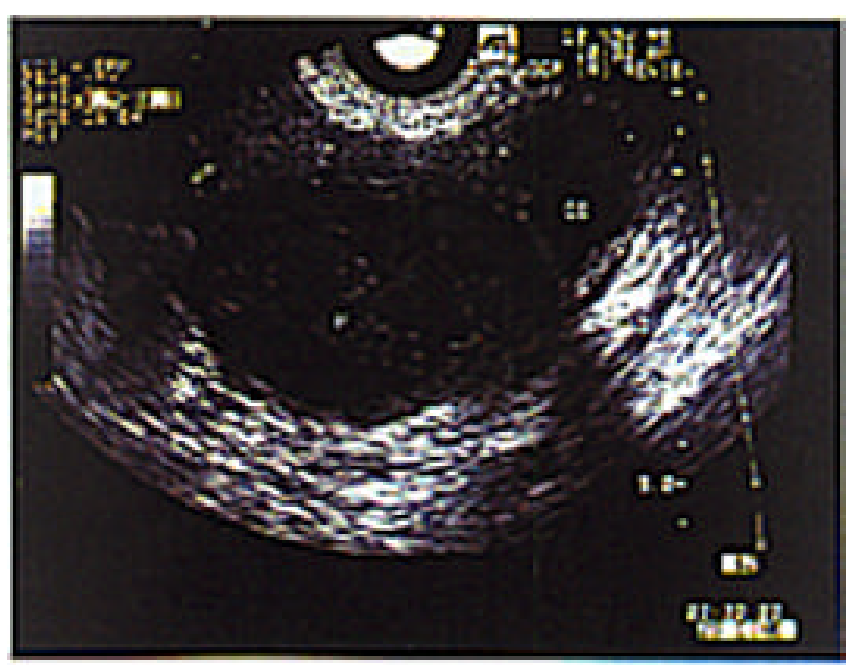

Figura 5 - Na ultra-sonografia transvaginal mostramos entre o útero e ovário esquerdo, formação complexa, heterogênea, típica de uma gravidez tubária com aspecto de hematossalpinge.

$\mathrm{Na}$ avaliação US pode-se determinar o diâmetro da massa anexial, sendo este um dos principais critérios de inclusão no grupo para tratamento medicamentoso. Desta forma, em nosso estudo as massas anexiais foram classificadas segundo seu diâmetro em: pequenas quando menores que $3,0 \mathrm{~cm}$, médias entre 3,0 e $4,0 \mathrm{~cm}$, e grandes com diâmetro de $4,1 \mathrm{~cm}$ a $5,0 \mathrm{~cm}$.

A quantidade de líquido livre na cavidade peritoneal foi observada e seu volume calculado conforme o trabalho de Nyberg e col. ${ }^{12}$, com algumas modificações por nós realizadas. Foram: consideramos como discreta (apenas em fundo de saco posterior), moderada (estendendo-se para região perianexial ipsilateral) e acentuada (extensão para regiões peri/supra-uterina e anexiais).

Na US com doppler colorido caracterizou-se como fluxo trofoblástico a presença de fluxo arterial de baixa resistência ao redor da massa, com índice de resistência menor que $0,45^{6}$. Como conseqüência dos estudos dopplervelocimétricos os casos foram classificados em três grupos: elevado, médio e baixo risco. O de elevado risco foi caracterizado por fluxo trofoblástico em mais de $2 / 3$ da massa anexial; de médio risco, quando comprometeu de $1 / 3$ a $2 / 3$ do anel tubário, como descreveram Atri et $\mathrm{al}^{2}$. Classificamos de baixo risco as seguintes situações: fluxos que acometiam menos de $1 / 3$ da massa anexial $^{2}$; ausência de vascularização ao redor da massa e presença de fluxos arteriais de resistência aumentada (IR maior que 0,5$)^{3}$; fluxos com morfologia bizarra quando se apresentavam com importante alargamento espectral, com tempos de aceleração longos e diástoles ausentes ou negativas ${ }^{6,14}$.

Firmado o diagnóstico de GE íntegra e 
incipiente, seguimos alguns critérios, antes de iniciar a terapêutica com MTX. Os critérios de inclusão foram a estabilidade hemodinâmica, o diâmetro da massa anexial menor ou igual a $5,0 \mathrm{~cm}$, desejo manifesto de gravidez futura pela paciente, e concordância em participar do estudo, através de consentimento escrito, em documento formulado e aprovado pela Comissão de Ética Médica da UNIFESP-EPM. Foram excluídas do tratamento medicamentoso da GE íntegra e incipiente as pacientes com necessidade de hemotransfusão, com reconhecida hipersensibilidade ao MTX, com evidências de doença hepática, renal ou supressão da medula óssea. Assim, não foram incluídas no protocolo aquelas com discrasias sangüineas, como leucopenia (menos que $2.000 \mathrm{cel} / \mathrm{mm}^{3}$ ) e trombocitopenia (menos que $100.000 / \mathrm{mm}^{3}$ ). Não participaram do protocolo as que apresentaram doença renal (creatinina maior que $1,5 \mathrm{mg} / \mathrm{dl}$ ). Acrescente-se, também, que não fizeram parte do estudo as mulheres com indicação de conduta expectante, isto é, os casos que apresentaram queda superior a $15 \%$ dos títulos de $\beta$-hCG num intervalo de 24 horas, sem terapêutica. Realizamos o procedimento de 2 dosagens consecutivas de $\beta$-hCG em 24 horas em todos os casos exceto na presença de embrião vivo ${ }^{2}$.

As pacientes cujos títulos de $\beta$-hCG dosados num intervalo de 24 horas que não apresentaram queda superior a $15 \%$, foram internadas no Hospital São Paulo da UNIFESP e tratadas com dose única de MTX $\left(50 \mathrm{mg} / \mathrm{m}^{2}\right.$ de superfície corporal por via intramuscular).

O acompanhamento se fez por meio de dosagens de $\beta$-hCG, realizadas no momento da ministração do MTX, no quarto e no sétimo dia após o emprego da droga. Consideramos relevantes estas três dosagens, já que pode ocorrer uma subida dos títulos de $\beta$-hCG nos primeiros 4 dias do tratamento, e isto não significa má evolução do caso, mas sim maior liberação de hormônio gonadotrófico na circulação secundária à ação do MTX. As que apresentaram redução maior que 15\% dos títulos de $\beta$-hCG, apurados no $4^{\circ}$ e no $7^{\circ}$ dia, tiveram alta hospitalar e seguimento ambulatorial com dosagens semanais de $\beta$-hCG, até que apresentassem títulos negativos. Quando no $7^{\circ}$ dia a queda foi menor que $15 \%$, ministramos, nova dose de MTX, em regime de internação, seguindo a mesma sistematização citada ${ }^{5,18}$. Os dados ultrasonográficos não foram utilizados como critérios de alta, já que a imagem ultra-sonografia pode persistir por algum tempo, mesmo após o declínio dos valores de $\beta-\mathrm{hCG}^{1,4,5}$.

O critério para classificação como de insucesso na ministração de MTX, na GE íntegra, baseou-se na persistência dos niveis de $\beta$-hCG, ou na redução menor que 15\%, após a primeira dose de MTX, ou na presença de sinais e sintomas clássicos denunciadores de ruptura tubária, apurados pela clínica e pela US transvaginal.

Após a alta hospitalar, as pacientes foram seguidas semanalmente em ambulatório realizando-se avaliações clínicas, ultrasonográficas e dosagens de $\beta$-hCG até que os títulos fossem negativos.

Consideramos como critério de sucesso do tratamento a queda maior que $15 \%$ dos títulos de $\beta$-hCG obtidos após a primeira dose de MTX, e que no acompanhamento semanal atingiram níveis inferiores a $5 \mathrm{mUI} / \mathrm{ml}$ (valor considerado negativo para este kit laboratorial).

As relações sexuais foram liberadas após o retorno dos valores de $\beta-\mathrm{hCG}$ a níveis prégravídicos. Orientava-se então o uso de anticoncepcional oral ou de método de barreira, por um periodo de 3 meses aproximadamente, pois neste prazo, geralmente, ocorre o desaparecimento da imagem ultra-sonográfica da massa anexial ${ }^{18}$.

Como controle, foi realizada a histerossalpingografia cerca de 3 meses após o tratamento. Posteriormente, as pacientes foram reavaliadas semestralmente, com anotação de nova gestação.

Foi elaborado um índice orientador do tratamento medicamentoso com MTX, baseado nos cinco parâmetros orientadores desta conduta: valores iniciais de $\beta-\mathrm{hCG}$, e por meio da US transvaginal, a análise do aspecto da imagem, do maior diâmetro da massa anexial, da dispersão do liquído livre e do "doppler" colorido.

Cada parâmetro recebeu pontuação de 0 a 2 , representando a nota zero elemento de mau prognóstico, a nota dois era destinada a parâmetros favoráveis ao tratamento medicamentoso com dose única de MTX, e a nota um para situações intermediárias (Tabela 1).

Tabela 1 - Índice orientador do tratamento sistêmico com dose única de MTX (50mg/m² IM).

\begin{tabular}{cccc}
\hline PARÂMETROS & \multicolumn{3}{c}{ PONTUAÇÃo } \\
& $\mathbf{0}$ & $\mathbf{1}$ & $\mathbf{2}$ \\
\hline B-hCG $(\mathbf{m U l} / \mathrm{ml})$ & $>5.000$ & $1.500-5.000$ & $<1.500$ \\
Aspecto da imagem & embrião vivo & anel tubário & hematossalpinge \\
Diâmetro da massa anexial em cm & $4,1-5,0$ & $3,0-4,0$ & $<3,0$ \\
Líquido livre & acentuado & moderado & discreto \\
doppler colorido & elevado risco & médio risco & baixo risco \\
\hline
\end{tabular}

A soma destes pontos foi comparada com os dados de sucesso ou fracasso, com o intuito de avaliar o valor destes parâmetros na indicação do tratamento medicamentoso.

Para a análise dos resultados foram utilizados 
testes não-paramétricos, levando-se em consideração a natureza das variáveis estudadas. Foram aplicados os seguintes testes com p de 0,05:

- Teste de Mann-Whitney para duas amostras independentes ${ }^{15}$, com a finalidade de comparar os grupos de pacientes com resposta favorável (sucesso) ou desfavorável (insucesso) em relação aos valores iniciais de $\beta$-hCG; diâmetro da massa anexial e nota do índice orientador com ou sem doppler colorido.

- Teste da partição do qui-quadrado para tabelas $2 \times \mathrm{N}$, com o objetivo de comparar os grupos de pacientes segundo os niveis séricos iniciais de ß-hCG $(<1.500,1.500-5.000,>5.000 \mathrm{mUI} / \mathrm{ml})$; aspecto da imagem (hematossalpinge, anel tubário, embrião vivo) e diâmetro da massa anexial $(<3,0,3,0-4,0,4,1-5,0$ cm), em relação ao sucesso ou insucesso do tratamento.

- Teste exato de Fisher para tabelas $2 \times 2^{15}$ com o objetivo de estudar as associações entre doppler colorido de alto risco e líquido livre discreto à US com sucesso ou insucesso do tratamento.

\section{Resultados}

De um grupo de 70 pacientes com diagnóstico de gravidez ectópica íntegra, 42 apresentaram os critérios para inclusão no grupo de tratamento sistêmico com MTX (50 mg/m² IM). Avaliamos todas as 5 variáveis do escore em 20 casos, e nos 22 restantes avaliamos todos os parâmetros propostos, exceto o doppler colorido.

Obtivemos sucesso com a dose única em 29 das 42 pacientes (69,0\%) e o insucesso ocorreu em $31,0 \%$ (13/42), como representado no Gráfico 1. Com uma segunda dose de MTX o índice de bons resultados se elevou para 78,6\% (33/42). Para cada parâmetro do índice orientador correlacionamos os seus resultados com o sucesso e insucesso do tratamento.

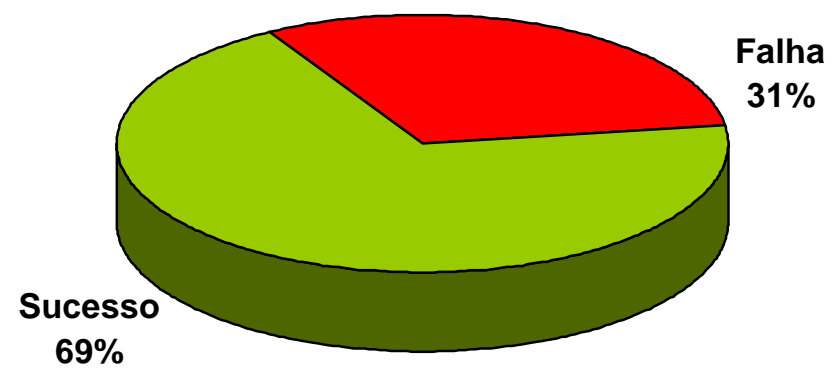

Gráfico 1 - Sucesso do tratamento da gravidez ectópica íntegra com dose única de Metotrexato $\left(50 \mathrm{mg} / \mathrm{m}^{2} \mathrm{IM}\right)$.
Com relação ao $\beta$-hCG, dividimos os seus valores no escore em títulos superiores a 5.000 $\mathrm{mUI} / \mathrm{ml}$, entre 1.500-5.000 mUI/ml, e inferiores a $1.500 \mathrm{mUI} / \mathrm{ml}$. Receberam pontuação de 0 a 2, em proporção inversa à titulação do hormônio. Para cada grupo correlacionamos os valores de $\beta$-hCG com os resultados do tratamento. Conforme verificamos na Tabela 2 , a maioria das pacientes apresentou títulos inferiores a $1.500 \mathrm{mUI} / \mathrm{ml}$ (24/42), denotando a freqüente associação de GE com níveis baixos de $\beta$-hCG. Em 21 casos obtivemos sucesso $(87,5 \%)$ e em 3 ocorreu o fracasso. Os valores intermediários de $\beta$-hCG (entre 1.500 e $5.000 \mathrm{mUI} / \mathrm{ml}$ ) foram observados em 9 pacientes, sendo que 6 destas $(66,7 \%)$ evoluíram com sucesso. Nove pacientes apresentaram valores acima de $5.000 \mathrm{mUI} / \mathrm{ml} \mathrm{e}$, destas, apenas $2(22,2 \%)$ evoluíram com sucesso. Na avaliação estátistica de qui-quadrado estes resultados são significantes $\left(\chi_{\text {calc }}^{2}=13,08^{*} ; \chi_{\text {crit }}^{2}=5,99\right)$. Após avaliarmos estes três grupos, observamos que títulos superiores a $5.000 \mathrm{mUI} / \mathrm{ml}$ estão associadas com o insucesso da terapêutica, ao passo que níveis inferiores a $1.500 \mathrm{mUI} / \mathrm{ml}$ estão associados com o sucesso.

Tabela 2 - Níveis séricos iniciais de ß-hCG em pacientes com gravidez ectópica íntegra tratadas com dose única de Metotrexato $\left(50 \mathrm{mg} / \mathrm{m}^{2} \mathrm{IM}\right)$.

\begin{tabular}{lcccc} 
NÍVEIS SÉRICOS & SUCESSO & INSUCESSO & TOTAL & $\%$ SUCESSO \\
\hline$<1.500$ & 21 & 3 & 24 & 87,5 \\
$1.500-5.000$ & 6 & 3 & 9 & 66,7 \\
$>5.000$ & 2 & 7 & 9 & 22,2 \\
\hline Total & 29 & 13 & 42 & 69,0 \\
\hline
\end{tabular}

Teste do qui-quadrado

$\chi_{\text {calc }}^{2}=13,08^{\star} \chi_{\text {crit }}^{2}=5,99$

Uma variável que consideramos relevante mas que nem sempre é valorizada nos relatos da literatura é o aspecto da imagem à US. Na Tabela 3 , relacionamos o aspecto da imagem com os resultados do tratamento. A grande maioria, ou seja, 23 pacientes apresentavam a imagem de hematossalpinge. Dos casos que evoluíram a contento, $22(95,6 \%)$ tinham este aspecto. O aspecto ultra-sonográfico de anel tubário, ou seja, saco gestacional sem ou com embrião sem atividade cardíaca, ocorreu em quinze casos, sendo que, das pacientes tratadas com êxito, 7 (46,7\%) apresentaram essa imagem à US. A imagem de embrião vivo foi vista na US em apenas 4 pacientes. Nenhuma evoluiu com sucesso, ou seja, em todos os casos com esse aspecto o tratamento medicamentoso foi um fracasso. Segundo análise estátistica de qui-quadrado, estes resultados foram significativos $\left(\chi_{\text {calc }}^{2}=20,06^{*} ; \chi_{\text {crit }}^{2}=5,99\right)$. 
Tabela 3 - Aspecto da imagem US das pacientes com gravidez ectópica íntegra tratadas com dose única de Metotrexato $\left(50 \mathrm{mg} / \mathrm{m}^{2} \mathrm{IM}\right)$.

\begin{tabular}{lcccc} 
IMAGEM & SUCESSO & INSUCESSO & TOTAL & $\%$ SUCESSO \\
\hline Hematossalpinge & $22^{*}$ & $1^{*}$ & 23 & $95,6^{*}$ \\
Anel tubário & 7 & 8 & 15 & 46,7 \\
Embrião vivo & $0^{*}$ & $4^{*}$ & 4 & 0,0 \\
\hline Total & 29 & 13 & 42 & 69,0 \\
\hline
\end{tabular}

Teste do qui-quadrado

$\chi_{\text {calc }}^{2}=20,06^{\star} \chi_{\text {crit }}^{2}=5,99$

A terceira variável analisada no índice que utilizamos é o diâmetro da massa anexial. Dividimos as massas em: diâmetro inferior a 3,0 $\mathrm{cm}$ (nota 2), entre 3,0 e 4,0 cm (nota 1) e acima de 4,0 até $5,0 \mathrm{~cm}$ (nota 0 ). Correlacionamos o tamanho do tumor anexial com o resultado da terapêutica. Podemos observar, na Tabela 4, a correlação do diâmetro da massa anexial com o resultado do tratamento. Nove pacientes apresentaram massas com menos de $3,0 \mathrm{~cm}$, oito $(88,9 \%)$ evoluíram com sucesso, e em apenas um caso ocorreu a falha terapêutica. As massas anexiais entre 3,0 e 4,0 $\mathrm{cm}$ ocorreram em dezesseis pacientes. Doze casos $(75,0 \%)$ evoluíram com sucesso. O diâmetro da massa superior a 4,0 até $5,0 \mathrm{~cm}$ foi observado em dezessete casos, sendo que nove $(52,9 \%)$ apresentaram bons resultados. O teste de quiquadrado não mostrou associação significante entre tamanho da massa anexial e o sucesso clínico $\left(\chi_{\text {calc }}^{2}=3,99 ; \chi_{\text {crit }}^{2}=5,99\right)$.

Tabela 4 - Diâmetro da massa anexial das pacientes com grazidez ectópica íntegra tratadas com dose única de Metotrexato $\left(50 \mathrm{mg} / \mathrm{m}^{2} \mathrm{IM}\right)$.

\begin{tabular}{lcccc} 
DIÂMETRO $(\mathrm{cm})$ & SUCESSO & INSUCESSO & TOTAL & \% SUCESSO \\
\hline$<3,0$ & 8 & 1 & 9 & 88,9 \\
$3,0-4,0$ & 12 & 4 & 16 & 75,0 \\
$>4,0$ & 9 & 8 & 17 & 52,9 \\
\hline Total & 29 & 13 & 42 & 69,0 \\
\hline
\end{tabular}

Teste do qui-quadrado

$\chi_{\text {calc }}^{2}=3,99 \quad \chi_{\text {crit }}^{2}=5,99$

Outro parâmetro considerado no índice que estamos propondo é a presença de líquido livre na cavidade peritoneal. É dado importante e no início do nosso trabalho, achávamos que a presença de hemoperitônio correspondia a ruptura tubária, apesar de Pellerito et al..$^{13}$ terem relatado a presença de líquido livre em 30\% dos casos de PE íntegra. Começamos, então, a notar, em muitos casos, a presença de líquido em pequena quantidade em pacientes assintomáticas e estáveis hemodina- micamente. Essas observações e os dados da literatura fizeram com que mudássemos de opinião, e não nos alarmássemos perante a presença de líquido livre na cavidade peritoneal, exceto na vigência de grande quantidade associada a instabilidade hemodinâmica. A análise dessa variável deve sempre ser acompanhada de dados clínicos da paciente, como a análise da cor das mucosas, o pulso e a pressão arterial.

Correlacionamos na Tabela 5 a presença de líquido livre com o sucesso e o insucesso do tratamento. A maioria das pacientes (37 casos) apresentavam pouco líquido livre, sendo que das $29(78,4 \%)$ tratadas com sucesso, todas tinham líquido em quantidade discreta. Das que evoluíram com insucesso, 8 apresentavam esse dado à US. Das pacientes com moderada e acentuada quantidade de líquido livre, nenhuma evoluiu com sucesso. Quando analisamos a variável líquido livre em quantidade discreta em confronto com a moderada e acentuada, apuramos pelo teste exato de Fisher $\left(p=0,0015^{*}\right.$ ou $\left.0,15 \%\right)$ associação significante entre sucesso terapêutico e líquido livre em quantidade discreta; por este motivo, atribuímos nota dois em nosso índice, ao passo que a presença de líquido livre em moderada e acentuada quantidade é um dado que reflete prognóstico desfavorável para esta terapêutica, e recebeu em nosso índice a nota um e zero, respectivamente.

Tabela 5 - Presença de líquido livre na cavidade peritoneal avaliado pela US nas pacientes com gravidez ectópica íntegra tratadas com dose única de Metotrexato $\left(50 \mathrm{mg} / \mathrm{m}^{2} \mathrm{IM}\right)$

\begin{tabular}{lcccc} 
LÍQUIDO LIVRE & \multicolumn{2}{c}{ TRATAMENTO } & TOTAL & $\%$ SUCESSO \\
& SUCESSO & INSUCESSO & & \\
\hline Discreto & $29^{*}$ & $8^{*}$ & 37 & $78,4^{*}$ \\
Moderado+acentuado & 0 & 5 & 5 & 0,0 \\
Total & 29 & 13 & 42 & 69,0 \\
\hline Teste exato de Fisher & & & & \\
$\mathbf{p}=\mathbf{0 , 0 0 1 5 ^ { \star }}$ ou $\mathbf{0 , 1 5 \%}$ & & & &
\end{tabular}

Por fim, o último parâmetro do escore por nós avaliado foi a US transvaginal com doppler colorido. Esse exame começou a ser realizado a partir do $23^{\circ}$ caso do nosso estudo, foi efetuado em 20 pacientes. A análise da vascularização da massa anexial com o intuito de se avaliar a presença ou não do fluxo trofoblástico e a sua intensidade é de grande importância na indicação do tratamento. O exame de doppler representa um retrato da gravidade do caso, enquanto aguardamos os resultados dos outro exames.

Na Tabela 6 correlacionamos os casos de doppler colorido com o resultado da terapêutica. Nessa tabela, confrontamos as pacientes com 
doppler de elevado risco com as de médio e baixo risco. O caso com doppler de elevado risco representaram 20,0\% (4/20) do total, sendo que um dos casos apresentava embrião vivo e os outro 3 anel tubário sem embrião. Nenhum caso com esta condição evoluiu com sucesso, o que representa 80,0\% (4/5) dos casos de insucesso. Estes dados submetidos ao Teste de Fisher foram significativos $\left(p=0,0010^{*}\right.$ ou $\left.0,10 \%\right)$. As situações com doppler de elevado risco, representaram um mau prognóstico para o tratamento sistêmico com MTX, e por isso lhe atribuímos nota zero em nosso indice.

Tabela 6 - US transvaginal com doppler colorido em pacientes com gravidez ectópica íntegra tratadas com dose única de Metotrexato $\left(50 \mathrm{mg} / \mathrm{m}^{2} \mathrm{IM}\right)$.

\begin{tabular}{lcccc}
\hline DOPPLER COLORIDO & \multicolumn{2}{c}{ TRATAMENTO } & TOTAL & \% SUCESSO \\
& SUCESSO & INSUCESSO & & \\
\hline Elevado risco & 0 & 4 & 4 & 0,0 \\
Baixo + Médio risco & 15 & 1 & 16 & 93,8 \\
Total & 15 & 5 & 20 & 75,0 \\
\hline $\mathbf{N}=\mathbf{2 0}$ & & & & \\
Teste exato de Fisher & & & & \\
$\mathbf{p}=\mathbf{0 , 0 0 1 0}$ ou $\mathbf{0 , 1 0 \%}$ & &
\end{tabular}

Na Tabela 7, realizamos a correlação da nota do índice prognóstico com doppler colorido com o sucesso e o insucesso da terapêutica. Das 20 pacientes submetidas ao escore, 75,0\% (15/20) evoluíram com sucesso e a média das notas foi 7,93, enquanto o insucesso ocorreu em 25,0\% $(5 / 20)$ e a média das notas foi 4,2 . Estes resultados submetidos a análise estátistica pelo teste de MannWhitney se revelaram significativos.

Com o intuito de estabelecermos uma nota de corte, ou seja, um valor abaixo do qual o tratamento medicamentoso seria contra-indicado, observamos as diversas notas de nosso índice orientador do tratamento sistêmico com MTX (Tabela 8). Em nossa casuística a nota de corte foi 5 , pois das pacientes com nota superior a $5,15 \mathrm{em}$ 16 evoluíram com sucesso $(93,75 \%)$, ao passo que as que apresentaram índices iguais ou inferiores a cinco estiveram todas relacionadas com o fracasso.

Com o intuito de avaliarmos a permeabilidade tubária após o tratamento medicamentoso, realizamos a histerossalpingografia em 21 pacientes. Apenas 3 casos demonstraram obstrução tubária e portanto o índice de permeabilidade tubária foi de $80,9 \%(17 / 21)$. Temos acompanhado as pacientes para verificar a ocorrência de gravidez sendo que até o momento 8 pacientes engravidaram.
Tabela 7 - Nota do índice orientador para o tratamento da gravidez ectópica íntegra com dose única de Metotrexato (50 mg/m² IM) Segundo o sucesso ou insucesso desta conduta.

SUCESSO INSUCESSO

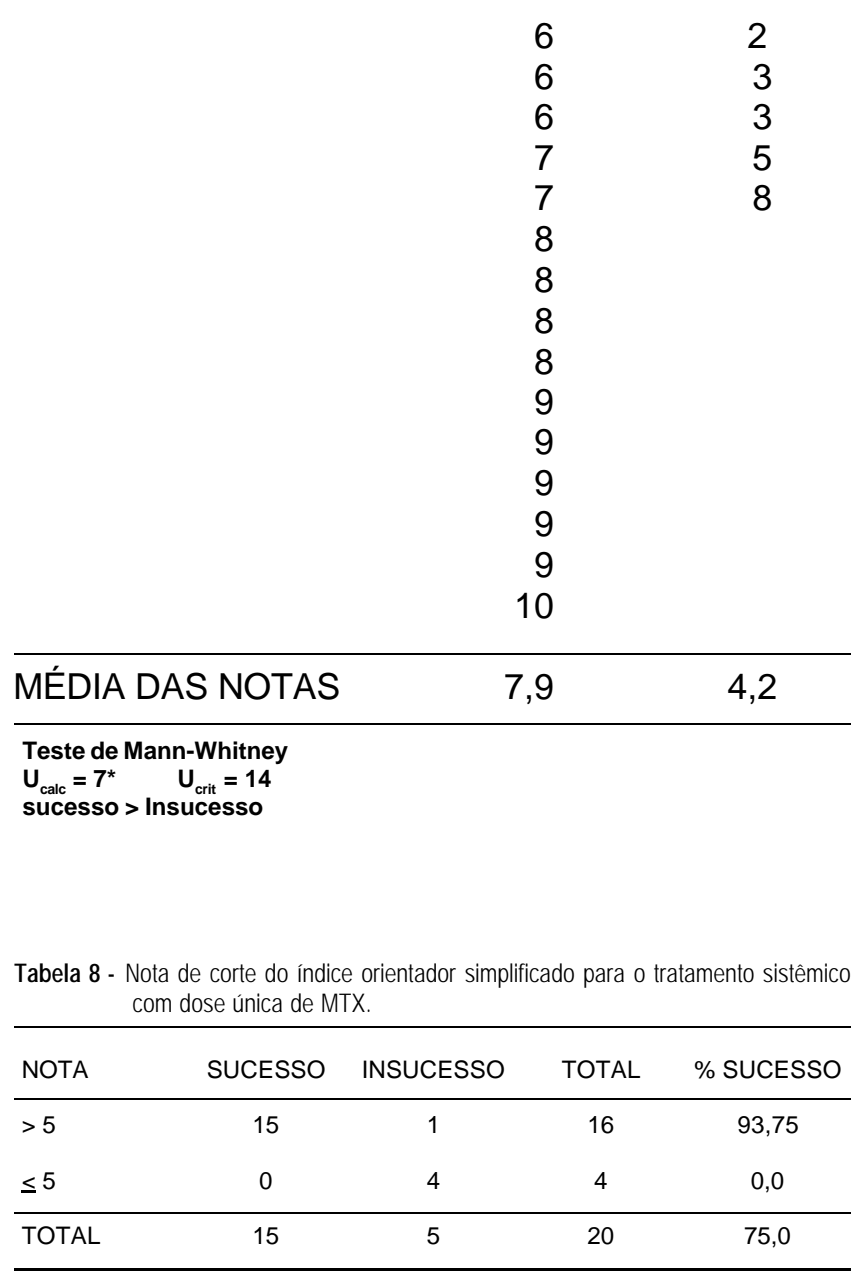

Teste exato de Fisher

$p=0,0010^{*}$ ou $0,10 \%$

\section{Discussão}

O objetivo do nosso trabalho foi elaborar um índice orientador do tratamento sistêmico com dose única de metotrexato $\left(50 \mathrm{mg} / \mathrm{m}^{2}\right)$, por via intramuscular, na gravidez ectópica íntegra. Cada parâmetro foi avaliado isoladamente. Ao analisarmos os valores de $\beta$-hCG observamos que títulos superiores a $5.000 \mathrm{mUI} / \mathrm{ml}$ cursam mais com o insucesso da terapêutica, ao passo que níveis inferiores a $1.500 \mathrm{mUI} / \mathrm{ml}$ estão associados com o sucesso. Demonstramos, portanto, que quanto menor o valor de $\beta$-hCG, melhor será a resposta ao tratamento medicamentoso. Em relação ao aspecto da imagem (hematossalpinge, anel tubário e embrião vivo), observa-se que quanto mais simular uma gravidez normal, pior será o 
prognóstico. O diâmetro máximo da massa anexial não diferiu muito dos casos de sucesso com os de insucesso, demonstrando que esse parâmetro valorizado pela literatura, no nosso estudo, não representou ser de importância para o prognóstico do tratamento. A maioria dos autores consideram $3,5 \mathrm{~cm}$ como tamanho máximo da massa anexial, obtendo como resultados do tratamento índices de sucessos que oscilaram de $78 \%$ e 96,7\% $\%^{9,10,16,17,18}$. Alguns, contudo, ampliaram esta indicação para tumorações maiores, de $3,5 \mathrm{~cm}$ até $5,0 \mathrm{~cm}$, conseguindo sucesso em $72,7 \%$ a $83 \%$ dos $\operatorname{casos}^{1,7,5,11}$

O parâmetro presença de líquido livre na cavidade peritoneal, quando em quantidade discreta ou ausente demonstrou ser situação favorável para o tratamento medicamentoso. A ultra-sonografia transvaginal com doppler colorido demonstrou em nosso estudo ser um dos parâmetros mais fiéis em relação ao prognóstico do tratamento.

A presença de doppler de alto risco esteve associado com o insucesso da terapêutica em 100\% dos casos, ao passo que $93,8 \%$ das pacientes com doppler de médio e baixo risco foram tratadas com sucesso. Estes dados sugerem que o exame de doppler isoladamente pode predizer, com segurança, os casos que devem ou não ser submetidos ao tratamento medicamentoso, já que demonstrou uma eficácia de 100\% em reconhecer os casos de insucesso e em 93,8\% os casos de sucesso. Por outro lado, em nossa amostra de 20 pacientes, todos os casos com doppler de elevado risco estavam avançados, ou seja, com massas anexiais com diâmetro superiores a $3,0 \mathrm{~cm}$ e títulos de $\beta$-hCG elevados ao redor de 5.000 mUI/ml. Não tivemos a oportunidade de diagnosticar nenhum caso com doppler de elevado risco na sua fase precoce, portanto, não sabemos como poderia evoluir uma paciente com esse dado numa fase inicial. Consideramos o doppler exame primordial e parâmetro de auxílio inegável no manejo do tratamento medicamentoso; todavia, acreditamos que a associação de outros parâmetros enriquece e nos traz dados mais fidedignos para orientação da terapêutica.

Das 20 pacientes submetidas ao escore, $75,0 \%(15 / 20)$ evoluíram com sucesso e a média das notas foi 7,93, ao passo que o insucesso ocorreu em $25,0 \%(5 / 20)$ e a média das notas foi 4,2 . As pacientes com notas inferiores a 5 evoluíram com insucesso (acrescida de 1 caso com nota 8). Saliente-se que todas as outras pacientes com notas maiores que cinco cursaram com o sucesso da terapêutica. Com a análise destes resultados concluímos que notas superiores a 5 apresentam melhor evolução ao tratamento medicamentoso.
Podemos sugerir ser este o valor de corte e quando o valores for inferior ou igual a 5 contra-indicamos esta terapêutica, optando pela cirurgia.

Quando se exclui a conduta expectante, isto é, quando não ocorre a queda maior que $15 \%$ dos títulos de $\beta$-hCG no intervalo de 24 horas, é de grande importância submeter a paciente ao índice orientador. Caso o escore seja superior a 5 podemos indicar o tratamento medicamentoso com dose única de metotrexato (50 $\mathrm{mg} / \mathrm{m}^{2} \mathrm{IM}$ ), com uma probabilidade grande de sucesso, minimizando a falha dessa terapêutica. Por outro lado, se o índice prognóstico for inferior ou igual a 5, o insucesso dessa conduta com uma única dose de MTX (50 $\mathrm{mg} / \mathrm{m}^{2} \mathrm{IM}$ ) é muito provável, e nessas circunstâncias, devemos optar por outras condutas resolutivas.

Ao término de nossas considerações, queremos enfatizar que nos últimos anos surgiram diversas terapêuticas relativas à gravidez ectópica, e o médico atento para as novas modalidades de tratamento, cuja indicação requer conhecimento de particularidades de cada uma delas, pode escolher para cada paciente a melhor conduta. Julgamos que o índice orientador por nós elaborado tem o intuito de nortear o tocoginecologista a eleger a conduta mais adequada, que proporciona os melhores resultados, fazendo com que as pacientes usufruam dos progressos da terapêutica em prol da sua saúde e do seu futuro reprodutivo.

\section{SUMMARY}

A prospective study was performed with 42 patients with unruptured ectopic pregnancy, which intended to elaborate an index to orient the systemic treatment with the administration of a single intramuscular dose of methotrexate $\left(50 \mathrm{mg} / \mathrm{m}^{2}\right)$. Patients were monitored with $\beta$ - $h C G$ titers on days 1,4 and 7 after the methotrexate. When the titers of beta-hCG declined more than $15 \%$, between days 4 and 7 after methotrexate, the patients were discharged and had an outpatient follow-up monitored with $\beta$ - $h$ CG titers weekly until the titers were less than $5 \mathrm{mIU} / \mathrm{ml}$, which represents success of the treatment. We prepared an index for the systemic treatment with methotrexate, with five parameters: (1) initial titers of $\beta$-hCG; (2) aspects of the image at ultrasound (hematosalpinx, gestational sac, live embryo); (3) size of the mass; (4) free fluid in cul-de-sac; (5) collor doppler. Each parameter received a grade from 0 to 2 . Grade 0 represented bad prognosis, favorable parameters received grade 2 and borderline parameters received grade one. The success rate with a single dose of methotrexate was $69.0 \%$ (29/42). The color doppler was performed in 20 of the 42 patients; in this group of 20 patients the success rate was $75.0 \%$ (15/20). In the 22 patients who were not submitted to the color doppler, the average grade of the score in the successful cases was 6.6, and in the unsuccessful it was 3.1. In the group who 
underwent the doppler (20 patients) the average was 7.9 in the successful cases and 4.2 in the cases that failed. In the present study the cut-off grade was 5, for most of the patients with grades above 5 had a successful treatment (15/16 $93.75 \%$ ), while grades equal or below 5 failed. The score will help to indicate the best cases for the medical treatment. We do not advise the treatment when the grade is equal or below 5. Therefore, we can predict a good evolution of the treatment when the grade is above five.

KEY WORDS: Ectopic pregnancy. Methotrexate. Chemotherapy.

\section{Referências bibliográficas}

1. Atri M, Bret PM, Tulandi T, Senterman M K. Ectopic pregnancy: evolution after treat with transvaginal MTX. Obstet Ultrassound 1992; 185: 749-53.

2. Atri M, Bret PM, Tulandi T. Spontaneous resolution of ectopic pregnancy: initial appearance and evolution at transvaginal US. Radiology 1993; 186: 83-6.

3. Bonilla-Musoles FM, Ballester MJ, Tarin JJ, Raga F, Osborne NG, Pellicer A. Does transvaginal Color Doppler Sonography differentiate between developing and involuting ectopic pregnancies? J Ultrasound Med 1995; 14:175-81.

4. Brown DL, Felker RE, Stovall TG, Emerson DS, Ling FW. Serial endovaginal sonography of ectopic pregnancies treated with methotrexate. Obstet Gynecol 1991; 77: 406-9.

5. Elito Junior J. O metotrexate no tratamento sistêmico da prenhez ectópica íntegra incipiente. São Paulo 1995. [Tese - Mestrado - Universidade Federal de São Paulo - Escola Paulista de Medicina].

6. Emerson DS, Cartier MS, Altieri LA, Felker RE, Smith WC, Stovall TG, Gray LA. Diagnostic efficacy of endovaginal color Doppler flow imaging in an ectopic pregnancy screening program. Radiology 1992; 183: 413-20.

7. Fernandez H, Benifla JA. Metotrexate treatment of ectopic pregnancy - 100 cases treated by primary transvaginal injection under sonographic control. Fertil Steril 1993; 59: 773-7.

8. Fernandez H, Bourget P. Les traitements médicaux de la GEU. Contracept Fertil Sex 1992; 20: 511-19.

9. Glock JL, Johnson JV, Brumsted JR. Efficacy and safety of single-dose systemic methotrexate in the treatment of ectopic pregnancy. Fertil Steril 1994; 62: 716-21.

10. Henry MA, Gentry WL. Single injection of methotrexate for treatment of ectopic pregnancies. Am J Obstet Gynecol 1994; 171: 1584-7.

11. Kojima E, Abe Y, Morita M, Ito M, Hirakawa S, Momose $\mathrm{K}$. The treatment of unrupture tubal pregnancy with intratubal methotrexate under laparoscopic control. Obstet Gynecol 1990, 75: 723-7.

12. Nyberg DA, Hughes MP, Mack LA, Wang KY. Extrauterine findings of ectopic pregnancy at transvaginal ultrasound: importance of echogenic fluid. Radiology 1991; 178: 823-6.

13. Pellerito JS, Taylor KJW, Quedens Case C, Hammers LW, Scoutt LM, Ramos IM, et al. Ectopic pregnancy: evaluation with endovaginal color flow imaging. Radiology 1992; 183: 407-11.

14. Reichmann AP, Elito Junior J, Lederman HM. Ectopic pregnancy: Further experience with the socalled "Bizarre waveforms". J Ultrasound Med 1997; 16: S79.

15. Siegel S. Estatística no parametrica. Mexico: Ed. Trillas 1975.

16. Stika CS, Anderson L, Frederiksen M. Single-dose methotrexate for the treatment of ectopic pregnancy: Northwestern Memorial Hospital threeyear experience. Am J Obstet Gynecol 1996; 174:1840-6.

17. Stovall TG, Ling GFW. Single dose of methotrexate: an expanded clinical trial. Am J Obstet Gynecol 1993; 168: 1759-65.

18. Stovall TG, Ling FW, Gray LA. Single dose methotrexate for treatment of ectopic pregnancy. Obstet Gynecol 1991; 77: 754-7.

19. Tanaka T, Hayashi H, Kutsuzawa T, Fujimoto S, Ichinoe K. Treatment of interstitial ectopic pregnancy with methotrexate: report of a successful case. Fertil Steril 1982; 37: 851-2. 